\title{
NOTE ON SETS OF POSITIVE MEASURE*
}

\section{BY HENRY BLUMBERG}

A recurring question concerning ( $L$-measurable) sets of positive measure is what properties they have in common with the linear interval. The following theorem is concerned with such a property, stated for sets of $n$-dimensional positive measure lying in euclidean $n$ space.

Theorem. Let $A_{1}, A_{2}, \cdots, A_{p}$ be $p$ sets of positive measure lying in euclidean $n$ space. Then there exist $p$ n-dimensional spheres $S_{1}, S_{2}, \cdots, S_{p}$ such that for every set of $p$ points $s_{\nu}$, $(\nu=1,2, \cdots, p)$, belonging respectively to these spheres, there exists a set of $p$ points $a_{\nu},(\nu=1,2, \cdots, p)$, lying respectively in $A_{1}$, $A_{2}, \cdots, A_{p}$, such that the sets $\left\{a_{\nu}\right\}$ and $\left\{s_{\nu}\right\}$ are congruent. Moreover, there exists a set of $p$ congruent spheres $S_{\nu}$ satisfying the condition just stated and a positive number $\delta$ such that for every selected $\left\{s_{\nu}\right\}$, with $s_{\nu}$ belonging to $S_{\nu}$, the associated $\left\{a_{\nu}\right\}$ may be so chosen that $a_{1}$ ranges over a set of measure $>\delta$.

Proof. Since $A_{\nu}$ is of positive measure, there is a sphere $S_{\nu}^{\prime}$ in which the relative measure of $A_{\nu}$ is greater than $1-\epsilon$, where $\epsilon$ is a given positive number less than 1 ; that is, $m\left(A_{\nu}, S_{\nu}^{\prime}\right) / m\left(S_{\nu}^{\prime}\right)$ $>1-\epsilon, m(A)$ standing for the measure of $A$. We may suppose, and we do so for simplicity of statement, that all the $S_{\nu}^{\prime}$, $(\nu=1, \cdots, p)$, are equal, and we denote their common measure by $\mu$, and their respective centers by $c_{\nu}$. Let $\rho$ be a positive number such that if a sphere of measure $\mu$ is translated a distance $<\rho$, the part belonging to the sphere in both positions is of measure $>(1-\epsilon) \mu$. Denote by $v_{\nu},(\nu=1, \cdots, p-1)$, the vector represented by the segment $c_{\nu} c_{\nu+1}$; and let $w_{\nu},(\nu=1, \cdots, p)$, be a given set of $n$-dimensional vectors, each of length $<\rho$. If a set $A$ (or point $a$ ) is given a displacement represented by the vector $\pm v$, we denote the set (or point) in its new position by $A \pm(v)$ (or $a \pm(v)$ ). Writing $A_{\nu} S_{\nu}^{\prime}=T^{(\nu)}$ and $T^{\prime}=T_{1}^{\prime}$, we set

$$
T_{1}^{\prime}+\left(v_{1}-w_{1}+w_{2}\right)=T_{2}^{\prime} ; T_{2}^{\prime} T^{\prime \prime}=T_{1}^{\prime \prime} ;
$$

\footnotetext{
* Presented to the Society, April 14, 1923.
} 


$$
\begin{gathered}
T_{1}^{\prime \prime}+\left(v_{2}-w_{2}+w_{3}\right)=T_{2}^{\prime} ; T_{2}^{\prime \prime} T^{\prime \prime \prime}=T_{1}^{\prime \prime \prime} ; \\
\text {. . . . . . . . . . . . . . } \\
T_{1}^{(p-1)}+\left(v_{p-1}-w_{p-1}+w_{p}\right)=T_{2}^{(p-1)} ; T_{2}^{(p-1)} T^{(p)}=T_{1}^{(p)} .
\end{gathered}
$$

For the measures of the $T_{\lambda}^{(\nu)}$ 's, we have the following inequalities: $m\left(T_{1}^{\prime}\right)>(1-\epsilon) \mu ; m\left(T_{1}^{\prime \prime}\right)>(1-4 \epsilon) \mu$, since $m\left(T^{\prime \prime}\right)>(1-\boldsymbol{\epsilon}) \mu$ and the lengths of $w_{1}$ and $w_{2}$ are less than $\rho ; m\left(T_{1}^{\prime \prime \prime}\right)>(1-7 \epsilon) \mu$, and so on. We may thus conclude that $m\left(T_{1}^{(p)}\right)>[1-(3 p-2) \epsilon] \mu$, which is positive if $\epsilon$ is taken small enough. We now define the spheres $S_{1}, \cdots, S_{p}$ of our theorem as of radii all less than $\rho$, and such that their respective centers $\gamma_{\nu}$ satisfy the relations: vector $\gamma_{\nu} \gamma_{\nu+1}=v_{\nu},(\nu=1, \cdots, p-1)$. If now $s_{\nu},(\nu=1, \cdots, p)$, is a point chosen from $S_{\nu}$, we let vector $\gamma_{\nu} s_{\nu}=w_{\nu}$. Let $a_{p}$ be a point of $T_{1}{ }^{(p)}$, which, as we have seen, is not empty if $\epsilon$ is sufficiently small. We then define $a_{p-1}, a_{p-2}, \cdots, a_{1}$ by the relation

$$
a_{\nu}=a_{\nu-1}+\left(v_{\nu-1}-w_{\nu-1}+w_{\nu}\right), \quad(\nu=2,3, \cdots, p) .
$$

Since $a_{p}$ belongs to $T_{1}{ }^{(p)}$, it belongs to $A_{p}$ and also to $T_{2}^{(p-1)}$; hence $a_{p-1}$ belongs to $T_{1}{ }^{(p-1)}$ and therefore to $A_{p-1}$ and $T_{2}{ }^{(p-2)}$; hence $a_{p-2}$ belongs to $T_{1}{ }^{(p-2)}$, and so on. We conclude that $a_{\nu},(\nu=1, \cdots, p)$, belongs to $A_{\nu}$. Since $s_{\nu}$ satisfies the equation $s_{\nu}=s_{\nu-1}+\left(v_{\nu-1}-w_{\nu-1}+w_{\nu}\right),(\nu=2, \cdots, p)$, we see that the sets $\left\{a_{\nu}\right\}$ and $\left\{s_{\nu}\right\}$ are congruent. Furthermore, since $a_{p}$ is an arbitrary point of $T_{1}{ }^{(p)}$, whose measure is arbitrarily near $\mu$, we can satisfy the last condition of our theorem by taking, for example, $\delta=\mu / 2$, if $\epsilon$ is small enough.

If, in particular, the $p$ given sets $A_{\nu}$ are identical, we may take the spheres $S_{\nu}^{\prime}$ as identical, thus reducing the vectors $v_{\nu}$ to zero. The spheres $S_{\nu}$ may therefore be taken as identical, and we have the following corollary.

Corollary. If $A$ is an n-dimensional set of positive measure, there exists an n-dimensional sphere $S$, such that for every finite subset of $S$ there is a congruent subset of $A$.

If $A$ is a one-dimensional set, we obtain the theorem of Steinhaus, ${ }^{*}$ that the set of distances between pairs of points of a

* Sur les distances des points, Fundamenta Mathematicae, vol. 1 (1920), p. 99. A simpler proof of this theorem, close in idea to our own, was published by Ruziewicz (after the present paper was read), Fundamenta Mathematicae, vol. 7 (1925), p. 141. 
(linear) set of positive measure contains an interval with 0 as left end point.

We have proved that if $S_{1}$ is a set of positive measure and $S_{2}$ a finite set, there is a subset of $S_{1}$ similar* to $S_{2}$. To what extent can the condition of finiteness of $S_{2}$ be modified if the theorem is to remain valid? Since every set of positive measure contains a perfect subset of positive measure, it follows that if every set of positive measure contains a set similar to $S_{2}$, it contains a set similar to $S_{2}+S_{2}^{\prime}$, where $S_{2}^{\prime}$ is the derivative of $S_{2}$. It thus suffices to restrict $S_{2}$ to being closed. Or we may restrict $S_{2}$ to being denumerable, since every set contains a denumerable subset which is dense in it. Not all sets of positive measure can contain a set similar to $S_{2}$ if $S_{2}$ is not non-dense. Since we naturally restrict $S_{2}$ to being bounded, we are led to ask: What bounded, non-dense sets $S_{2}$ are such that every set of positive measure contains a set similar to $S_{2}$ ? That this property is not shared by every bounded, non-dense $S_{2}$, and therefore not by every bounded, non-dense, denumerable set, is shown by the following fact.

THEOREM. If $S_{1}$ is a given bounded, non-dense, perfect set, there exists a perfect set $S_{2}$ of zero measure such that no subset of $S_{1}$ is similar to $S_{2}$.

While this theorem is meant to refer to $n$-dimensional sets, we assume in the proof that $S_{1}$ and $S_{2}$ are linear sets, there being no essential difference in the argument for $n$-dimensional sets. We suppose, as we may, that the given set $S_{1}$ lies in the interval $(0,1)=I$. Let $C\left(S_{1}\right)$ be the complement of $S_{1}$ in $I ; \Delta$ a variable subinterval of $I ; \mu_{1}(\Delta)$ the ratio of the maximum length of a connected portion of $C\left(S_{1}\right)$ in $\Delta$ to the length of $\Delta$; and $\sigma_{1}(h)$, for $h$ a given positive number, the greatest lower bound of $\mu_{1}(\Delta)$ for all subintervals $\Delta$ of $I$ of length $h$. Then $\sigma_{1}(h)$ is a positive, continuous function of $h$. The perfect set $S_{2}$ will be defined as the complement in $I$ of the set of intervals $\Delta_{n i}$, which are defined as follows: Insert in $I$ a set of equally spaced intervals $\Delta_{1 i},\left(i=1,2, \cdots, m_{1}\right)$, of equal length $l_{1}$, such that $m_{1} l_{1}=1 / 2$, the equality of spacing being understood in the sense that the

* We are using "similar" in the ordinary euclidean sense of the existence of a biunique correspondence with invariant ratio of distances. 
space between any two adjacent $\Delta_{1 i}$ shall be equal to the spaces between 0,1 and the first, last $\Delta_{1 i}$, respectively; moreover, $m_{1}$ is to be so large that $\sigma_{2}\left(\lambda_{1}\right)<\sigma_{1}\left(\lambda_{1}\right)$, where $\lambda_{1}=1$, and $\sigma_{2}$ has the same meaning for the set $S_{2}$, now being defined, as $\sigma_{1}$ for $S_{1}$. Similarly insert in each of the intervals $\Delta_{1 i}^{\prime},\left(i=1,2, \cdots, m_{1}+1\right)$, of length $l_{1}^{\prime}$, that are complementary to the $\Delta_{1 i}$ the same number of equally-spaced intervals $\Delta_{2 i},\left(i=1,2, \cdots, m_{2}\right)$, of equal length $l_{2}$, where $m_{2}$ signifies the total number of the $\Delta_{2 i}$ in all the $\Delta_{1 i}^{\prime} ;$ moreover, the $\Delta_{2 i}$ are to be such that $m_{2} l_{2}=1 / 4$, and $m_{2}$ so large that $\sigma_{2}\left(t \lambda_{2}\right)<\sigma_{1}\left(\lambda_{2}\right)$ for $1 \leqq t \leqq 2$, where $2 \lambda_{2}=l_{1}^{\prime}$. In general, let $\left\{\Delta_{n-1, i}^{\prime}\right\}$ be the set of intervals of length $l_{n-1}^{\prime}$, complementary to the set of all $\Delta_{\nu i}, \nu \leqq n-1$. Insert in each $\Delta_{n-1, i}^{\prime}$ the same number of equally spaced intervals $\Delta_{n i}$ of equal length $l_{n}$, such that $m_{n} l_{n}=1 / 2^{n}, m_{n}$ being the total number of intervals $\Delta_{n i}$ and $m_{n}$ so large that $\sigma_{2}\left(t \lambda_{n}\right)<\sigma_{1}\left(\lambda_{n}\right)$ for $1 \leqq t \leqq n$, where $n \lambda_{n}=l_{n-1}$. Suppose now that $S_{3}$ is any set whatsoever lying in $I$ and similar to $S_{2}$; then $S_{3}$ cannot lie in $S_{1}$. For let $k$ be the ratio of corresponding lengths in $S_{2}$ and $S_{3}$, and $n$ an integer greater than $k$. If $\epsilon$ is a given positive number, we can find an interval $\Delta$ of length $\lambda_{n}$ lying between two points of $S_{3}$, and such that $\left|\mu_{3}(\Delta)-\sigma_{2}\left(k \lambda_{n}\right)\right|<\epsilon, \mu_{3}$ having the same meaning for $S_{3}$ as $\mu_{1}$ for $S_{1}$. Hence, on account of the inequality $\sigma_{2}\left(k \lambda_{n}\right)<\sigma_{1}\left(\lambda_{n}\right)$, we conclude that $\mu_{3}(\Delta)<\sigma_{1}\left(\lambda_{n}\right) \leqq \mu_{1}(\Delta)$, if $\epsilon$ is small enough. That is to say, the maximum length of a connected portion of $C\left(S_{3}\right) \Delta$ is less than such maximum length for $C\left(S_{1}\right) \Delta$, and therefore $S_{3}$ cannot lie in $S_{1}$.

The Ohio Srate University 